\title{
Failure Precursors for Insulated Gate Bipolar Transistors (IGBTs)
}

\author{
Nishad Patil ${ }^{1}$, Diganta Das ${ }^{1}$, Kai Goebel ${ }^{2}$ and Michael Pecht ${ }^{1}$ \\ ${ }^{1}$ Center for Advanced Life Cycle Engineering (CALCE) \\ University of Maryland \\ College Park, MD 20742, USA \\ ${ }^{2}$ NASA Ames Research Center \\ Moffett Field, CA 94035, USA
}

\begin{abstract}
Failure precursors indicate changes in a measured variable that can be associated with impending failure. By identifying precursors to failure and by monitoring them, system failures can be predicted and actions can be taken to mitigate their effects. In this study, three potential failure precursor candidates, threshold voltage, transconductance, and collector-emitter ON voltage, are evaluated for insulated gate bipolar transistors (IGBTs). Based on the failure causes determined by the failure modes, mechanisms and effects analysis (FMMEA), IGBTs are aged using electrical-thermal stresses. The three failure precursor candidates of aged IGBTs are compared with new IGBTs under a temperature range of $25-200^{\circ} \mathrm{C}$. The trends in the three electrical parameters with changes in temperature are correlated to device degradation. A methodology is presented for validating these precursors for IGBT prognostics using a hybrid approach.
\end{abstract}

Keywords: Failure precursors, IGBTs, prognostics

Failure precursors indicate changes in a measured variable that can be associated with impending failure [1]. A systematic method for failure precursor selection is through the use of the failure modes, mechanisms, and effects analysis (FMMEA) [2]. By identifying and monitoring the precursors, the extent of deviation or degradation from expected normal operating conditions can be assessed to provide advanced warning of failures.

In this article, three electrical parameters of the insulated gate bipolar transistors (IGBTs), threshold voltage, transconductance, and collector-emitter ON voltage, are evaluated for IGBTs aged by electrical-thermal stresses. The aged IGBT parts are compared with new parts in a temperature range of $25-200^{\circ} \mathrm{C}$ to assess their potential as precursor candidates.

\section{INTRODUCTION}

Insulated Gate Bipolar Transistors (IGBTs) are used in switching applications for automobile and train traction motors, in high voltage power supplies, and in aerospace applications such as switch mode power supplies (SMPS) to regulate DC voltage. The failure of these switches can reduce the efficiency of the system or lead to system failure. IGBTs have switching characteristics similar to a metal oxide semiconductor field effect transistor (MOSFET), as well as high current and voltage capabilities of a bipolar junction transistor (BJT). The structure of an IGBT is similar to that of a vertical diffusion power MOSFET (VDMOS) [3] except for an additional $\mathrm{p}^{+}$layer above the collector as seen in Figure 1. The main characteristic of the vertical configuration is that the collector (drain) forms the bottom of the device while the emitter (source) region remains the same as a traditional MOSFET in this configuration. Figure 1 represents the schematic structure of the device used in this study. The majority carriers in this device are electrons. The additional $\mathrm{p}^{+}$layer in the IGBT acts as a source of holes that are injected into the body (n- region) during operation.

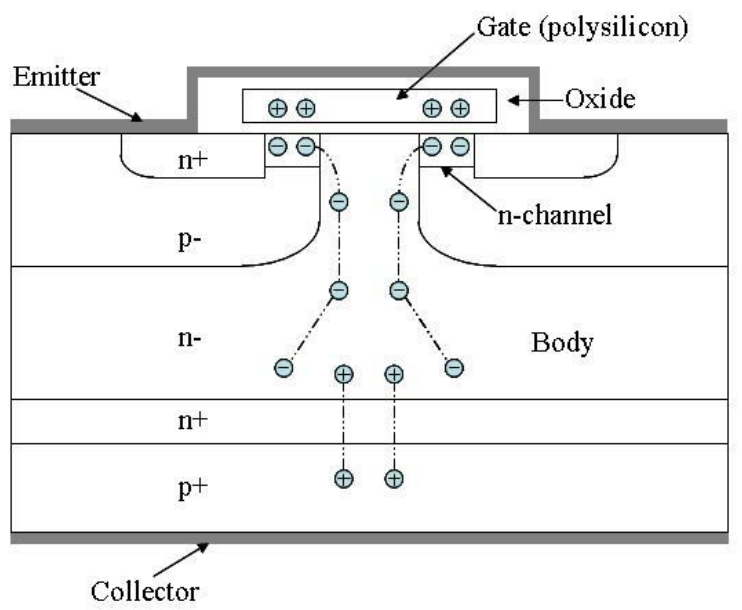

Figure 1: Schematic of IGBT Operation 
Applying a positive voltage to the gate switches on the device when an inversion layer (i.e., a conductive channel) is created in the $\mathrm{p}$ - region just below the gate oxide, allowing for electron flow. Electrons flow from the emitter through the conductive channel to the collector terminal. As IGBTs are switched on by voltage rather than current, they have faster switching speeds in comparison to the BJT. A positive voltage applied to the collector with the emitter at ground causes injection of positive carriers from the $\mathrm{p}^{+}$layer, which contacts the collector, into the body. This leads to excess charges in the body of the device reducing body resistance. The process of reducing body resistance with increasing carrier concentration is called conductivity modulation. This process allows the IGBT to have a lower on-resistance compared to the power MOSFET. The punch-through IGBT used in this study has an additional $n+$ layer, called the buffer layer, above the $\mathrm{p}^{+}$layer that contacts the collector terminal. When the IGBT is turned off, the positive carriers injected from the $\mathrm{p}+$ layer remain in the body of the device. The additional $n+$ layer in the punch-through configuration allows for faster evacuation of the stored positive carriers in the body. This reduces the current fall time, turn-off time and switching loss of the IGBT.

\section{FAILURE MODES MECHANISMS AND EFFECTS ANALYSIS (FMMEA) OF IGBTS}

FMMEA is a methodology to identify potential failure mechanisms and models for all potential failure modes, and to prioritize failure mechanisms. The output of the FMMEA process is a list of critical failure mechanisms that help us identify the precursors to monitor and the relevant physics of failure models to use; enabling the prediction of the component's remaining useful life. In the hybrid prognostic methodology being used in this research (described in the hybrid PHM methodology section); FMMEA is the first step in the analysis.

The failure modes for the IGBT are in the form of degradation of certain key electrical parameters (e.g., leakage current, threshold voltage) or the loss of functionality (inability to turn-off). The failure causes can be due to environmental conditions or operating conditions. For example, failures could be caused by high humidity, leading to corrosion of electrical contacts. Failures could also be caused by large temperature variations leading to unequal expansion within different materials that make up the device resulting in interface degradation. High temperatures during operation could lead to an accumulation of defects in the gate oxide leading to its breakdown by the time dependent dielectric breakdown mechanism. The FMMEA performed in this study is limited to die level failures only.
Critical transistor failure mechanisms include hot electrons and gate oxide breakdown. Gate oxide breakdown can result in excessive leakage current, increased standby power, and an increase in transistor response time. The FMMEA for IGBTs is illustrated in Figure 2. In this figure, one critical failure mechanism for each site is listed.

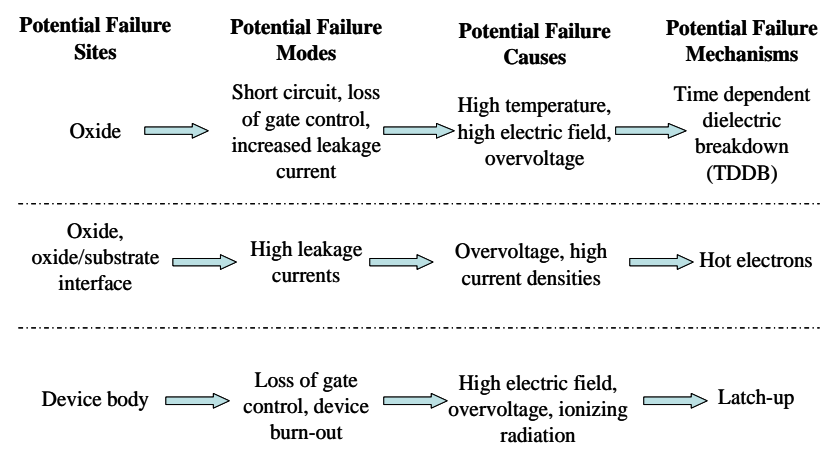

Figure 2: FMMEA of IGBTs

The potential failure causes for all the mechanisms as shown in Figure 2 involve high electric fields. In addition to high electric fields, oxide breakdown also involves high temperature. The IGBTs are therefore subjected to electrical and thermal stresses to degrade the parts through failure mechanisms of interest to identify precursors to failure.

\section{ELECTRICAL-THERMAL AGING}

Accelerated aging on International Rectifier IRG4BC30KD insulated gate bipolar transistors (IGBTs) used in this study was performed by Impact Technologies. The aging was performed by controlling the duty cycle and increasing the collector-emitter voltage. The temperature at the transistor case was increased up to $315^{\circ} \mathrm{C}$ by this process. The collector-emitter voltage was increased from 4 to 6.5 volts in a time period of forty five minutes. The collector-emitter leakage current was continuously monitored during the test. When latching of the IGBT was imminent, as was evidenced by the loss of gate control and increased collector-emitter leakage, the aging process was stopped. The IGBTs did not lose functionality due to this stressing and returned to a functional state after the aging exposure.

\section{DEGRADATION ANALYSIS}

Degradation analysis was performed to determine the effects of electrical-thermal aging on the package of the IGBTs. A non-destructive technique called scanning acoustic microscopy (SAM) was used. Scanning acoustic 
microscopy analysis is used to detect delaminations and voids in plastic encapsulated microcircuits. This technique is sensitive to submicron thickness delaminations.

For the SAM analysis, the TO-220 package was placed with the heat-sink facing the transducer. A $35 \mathrm{MHz}$ transducer was used in the pulse-echo mode to obtain the C-scan image of the die-attach interface to detect voids and delaminations in the die-attach layer. Voids within the die-attach and delamination between the die and the dieattach appear as bright white areas in the image.

Two aged parts labeled “a1" and “a2" were selected for analysis. The C-scan images of these parts are shown in Figure 3. This device is a co-pack wherein the IGBT (large die on the left) and a free-wheeling diode (smaller die on the right) are present in a single package. Part "a1" has significantly more damage in the IGBT die-attach in comparison to part “a2”.

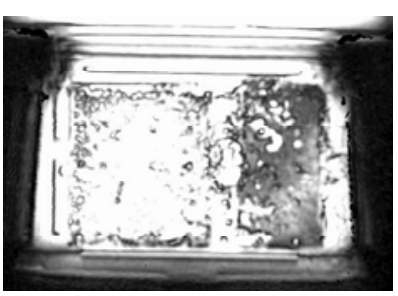

Part a1

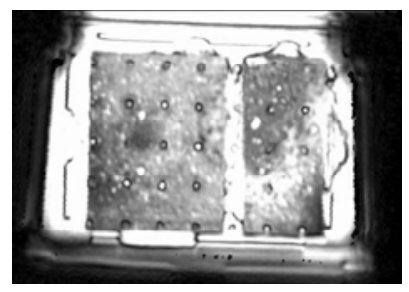

Part a2

Figure 3: CSAM images of IGBT parts "a1" and "a2"

X-ray images of the aged parts are shown in Figure 4. The $\mathrm{X}$-ray images correlate with the CSAM images as observed by comparing the parts "a1" and "a2" in Figure 3 and Figure 4. The images show varying levels of die attach degradation in the other three parts too.

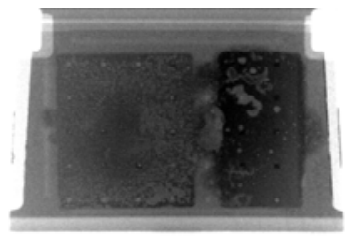

a1

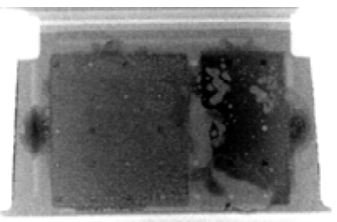

a3

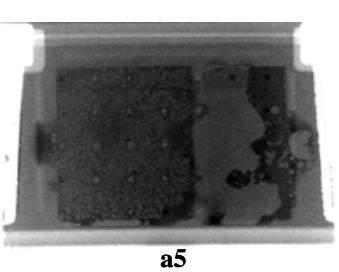

Figure 4: X-ray images of aged IGBT parts
The die-attach degradation could have occured as a result of thermal gradients within the device during aging. In an earlier study, thermal flows were modeled as one dimensional heat transfer through the silicon chip and dieattach to the heat sink and the simulation results indicated that very high temperatures occur in the silicon chip, whereas temperature variations in the solder die-attach remain relatively low [4]. This thermal gradient could lead to the die-attach degradation observed in the IGBTs. The delaminations/voids in the die-attach can be considered as measure of electrical-thermal aging of the part.

\section{EFFECT OF TEMPERATURE ON NEW AND ELECTRICALLY-THERMALLY AGED IGBTs}

The effects of temperature on the behavior of the three electrical parameters of IRG4BC30KD IGBTs were evaluated. Five new IGBTs were used in this study to form a baseline for healthy behavior. Due to statistical variations in the fabrication processes such as in the channel doping and oxide charge, the electrical parameters will be spread in a distribution, even in transistors that belong to the same production lot [5]. Five new parts were used in the analysis to capture the nature and extent of this spread. The behavior of five aged parts with different levels of damage was then examined with that of the new parts. The Temptronic thermal stream generator was used to subject the parts to temperatures ranging from $25^{\circ} \mathrm{C}$ to $200^{\circ} \mathrm{C}$ in steps of $25^{\circ} \mathrm{C}$. This analysis was performed to determine if the aged parts behave differently in comparison to new parts over a temperature range. At every temperature step, three measurements were taken for each electrical parameter. The mean of the three measurements was used to plot the variation in electrical parameters with temperature. All measurements were performed using the Tektronix high power curve tracer. The results obtained for all measurements have differences from the datasheet values due to the additonal resistance caused by the wiring from the curve tracer to the thermal stream generator.

\section{Threshold Voltage}

The threshold voltage is the gate voltage at which the IGBT turns on and collector current begins to flow. IGBTs have a negative temperature co-efficient for the threshold voltage as seen in Figure 5. As observed, the threshold voltage drops as temperature increases. The increase in temperature leads to decrease in the band-gap of the silicon, which reduces the threshold voltage. It is therefore easier to turn-on the IGBT at higher ambient temperatures.

The threshold voltage reduces with increase in temperature for aged as well as new parts. From the experiments, it is observed that the aged parts have a 
higher threshold voltage than the new parts across all temperatures. The difference between the average threshold of the new parts and the aged part "a1" when measured at $25^{\circ} \mathrm{C}$ is $10.5 \%$ and this difference reduces to $9.7 \%$ at $200^{\circ} \mathrm{C}$. This suggests that temperature affects new and aged parts in the same manner.

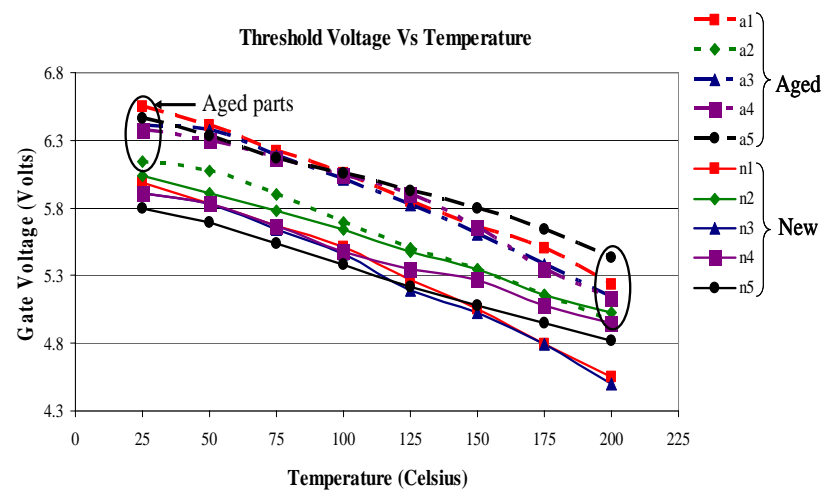

Figure 5: Threshold voltage variation with temperature

\section{Transconductance}

Transconductance is the gain of the transistor and refers to the magnitude of output current (collector current) obtained for a given input voltage (gate voltage). IGBTs have a negative temperature co-efficient for the transconductance as seen in Figure 6.

Therefore, the gain of the IGBT reduces with increase in temperature, and the output current from the transistor reduces for a given gate voltage. From the experiments, it is observed that the transconductance for the aged parts is higher compared to the new parts. The difference between the average transconductance of new parts and the aged part "a1" at room temperature is $8.2 \%$ and the difference reduces to $5.6 \%$ at $200^{\circ} \mathrm{C}$.

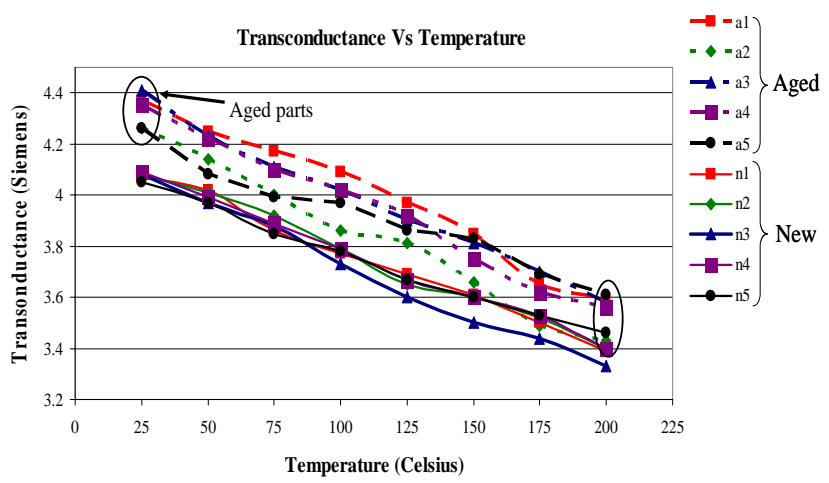

Figure 6: Transconductance variation with temperature

\section{Collector Emitter ON Voltage}

IGBTs have a positive temperature co-efficient for the collector emitter ON voltage at high collector currents [7] as seen in Figure 7. With an increase in temperature, the collector-emitter voltage value increases. Thus, with an increase in temperature, the IGBT output current reduces due to increased resistance. From the experiments, it is observed that the collector-emitter ON voltage for the new parts is higher in comparison to the aged parts. The difference between the average collector emitter $\mathrm{ON}$ voltage difference and the aged part "a 1 " is $9.9 \%$ at $25^{\circ} \mathrm{C}$ and the difference increases to $12.8 \%$ at $200^{\circ} \mathrm{C}$.

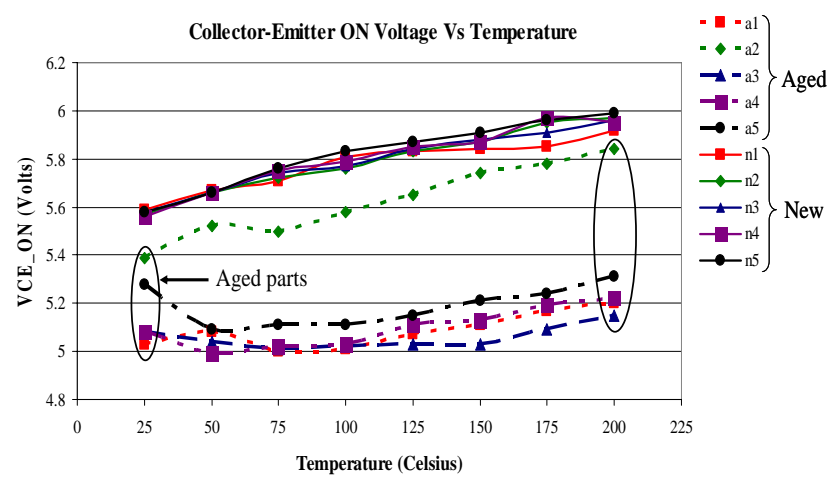

Figure 7: Collector-emitter ON voltage variation with temperature

\section{DISCUSSION}

From the electrical-thermal stress test results, we observe that the threshold voltage in aged parts is higher than in the new parts. The positive shifts in threshold voltage are consistent with the presence of trapped electrons in the gate oxide. Holes trapped in the gate oxide lead to a negative threshold shift while trapped electrons lead to a positive threshold shift.

The tranconductance is observed to be higher in the aged parts compared to the new parts. This indicates that the aged parts output more collector current for a given gate voltage as compared to the new parts. This is also verified by the collector-emitter ON voltage results which show that the aged parts have lower resistance compared to the new parts.

The delaminations/voids observed in the X-ray analysis of the aged parts correlate to the trends observed in the evolution of the three parameters. For the collector-emitter ON voltage results shown in Figure 7, the aged part "a2" which has a less degraded die-attach in comparison to the other aged parts, follows the trend of the new parts. This suggests that the die-attach degradation is correlated to the collector-emitter ON voltage parameter.

The higher transconductance and lower collector-emitter ON voltages of the aged parts can be explained by noting that the transconductance and the collecter-emitter ON voltages are measured at a gate voltage of $15 \mathrm{~V}$ and 
collector current of $16 \mathrm{~A}$. At these relatively high currents, the degraded die-attach may lead to an increased temperature at the p-n junction above the collector. As the p-n junction has a negative temperature coefficient of resistance [6], an increase in the temperature at this junction will result in lowered resistance. This could explain the observed low resistance in the aged parts compared to the new parts.

This hypothesis is supported by the I-V charactersitics measured at room temperature for gate voltages between 10-11.5 V for an electrically-thermally aged part "a1" and new part " $n 1$ " as shown in Figure 8. At these gate voltages, high currents are output by the transistor. As observed, the collector current for the aged part is much higher than the new part for a given gate and a given collector-emitter voltage in the linear region. The linear region is defined as the region where the collector current is proportional to the collector-emitter voltage. The results as seen in Figure 8 show that aged parts have lower resistances at high currents.

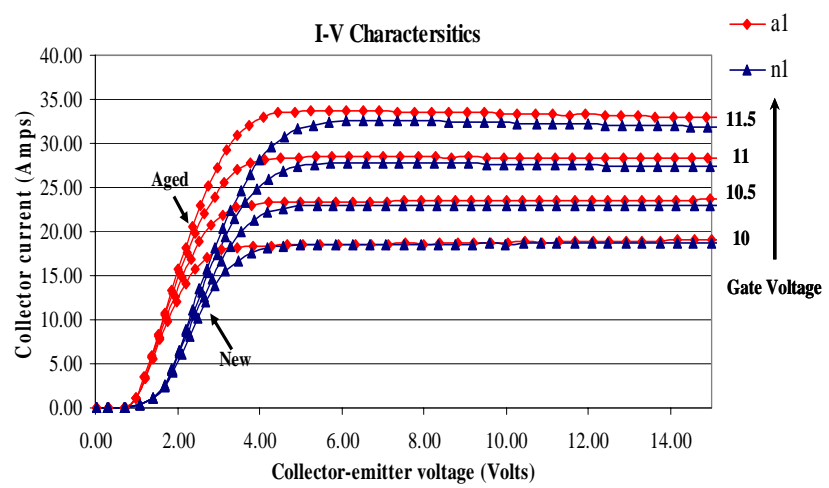

Figure 8: I-V curves for aged and new IGBTs under high gate voltages

One would expect that at lower gate voltages and lower currents, the impact of the degraded die attach would not be as significant. The I-V charactersitics measured at low gate voltages support this view as shown in Figure 9.

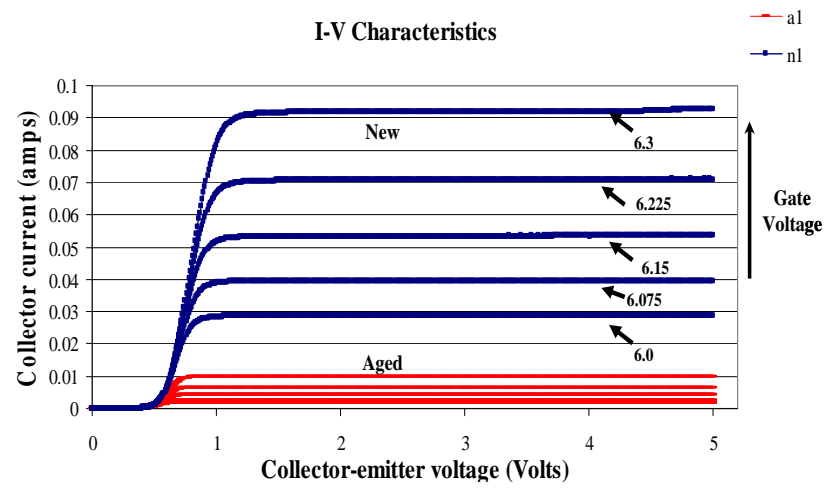

Figure 9: I-V curves for aged and new IGBTs under low gate voltages
As seen, at lower gate voltages, the aged part has a lower collector current compared to the new part. The higher resistance of the aged part can be attributed to the trapped charges in the oxide that cause increased channel resistance. At these low currents, the degraded die-attach has negligible impact.

From the discussion, it can be concluded that two mechanisms are in operation; the oxide damage that affects the threshold voltage, and the die-attach degradation which affects the transconductance and collector-emitter ON voltage.

\section{HYBRID PHM METHODOLOGY}

The hybrid PHM approach involves identifying precursor parameters, determining their correlation with system performance parameters, continuous monitoring of product health, detecting failure trends, and estimating the time to failure. The first step in this process is to perform the FMMEA of the device and identify precursors to failure. Next, parameters that can be monitored in application are selected. These parameters are continuously monitored and compared with the baseline of healthy devices. An alarm is generated when anomalous behavior is detected. The parameters that contribute significantly to the anomaly are isolated. The isolated parameters and monitored environmental loads form the input to the physics of failure (PoF) models that are subsequently used for estimating remaining useful life. Our hybrid PHM methodology for IGBT prognostics is shown in Figure 10.

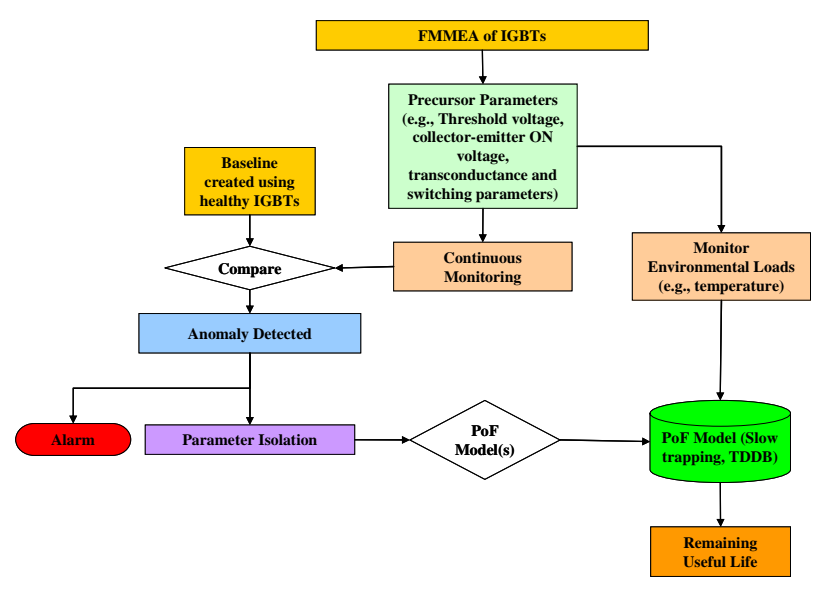

Figure 10: Hybrid PHM methedology for IGBTs

In this study, the first two steps in the Hybrid PHM approach have been performed. One of the three parameters monitored in this study, the transconductance, is a dynamic parameter. Dynamic parameters are suitable for in-situ monitoring. The oxide damage that affects the 
threshold voltage also affects the switching parameters, such as turn-on and turn-off times [7]. The switching parameters are also dynamic parameters and are therefore potential failure precursors that are suitable for continuous monitoring in application. The precursor data obtained from monitoring these parameters will form the input to the PoF models for estimation of time to failure. For the IGBTs, we expect to use the PoF failure models for slow trapping [8] and time dependent dielectric breakdown [9] to account for trapped charges in the gate oxide.

\section{SUMMARY AND CONTINUING WORK}

The electrical-thermal stresses used to age the IGBTs leads to trapped electrons in the gate oxide resulting in an increase in the threshold voltage of the aged parts. The observed increase in the transconductance (gain) and reduction in collector-emitter ON voltage of the electrically-thermally aged parts is possibly a result of the observed degradation in the die-attach.

The threshold voltage, transconductance and collectoremitter ON voltage are precursors to IGBT failure as all three parameters show changes with increased degradation. Further, the trends in the evolution of parameters helps identify the failure mechanism in operation, as observed by the changes in the threshold voltage (trapped charges in the gate oxide) and collectoremitter ON voltage (degraded die-attach). The switching parameters such as turn-on delay time and turn-off fall time which are directly affected by gate oxide damage, are potential precursors to IGBT failure. The transconductance and switching parameters are dynamic parameters that can be continuously monitored in application.

A hybrid PHM approach, where these precursors are monitored in-situ and precursor trending data are input into Physics of Failure (PoF) models, will allow for anomaly detection and prediction of remaining life of these devices.

\section{ACKNOWLEDGEMENTS}

This work is sponsored by the members of the CALCE Prognostics and Health Management Consortium at the University of Maryland, College Park. The authors thank Impact technologies for providing information on the electrical-thermal aging process and for providing the aged parts used in this study.

\section{REFERENCES}

[1] N. Vichare and M. Pecht, "Prognostics and Health Management of Electronics," IEEE Transactions on Components and Packaging Technologies, 29, 2006, 222229,

[2] M. Pecht, "Prognostics and Health Management of Electronics,” Wiley-Interscience, 2008.

[3] J. Baliga, "Power Semiconductor Devices,” PWS Publishing Company, 1996.

[4] Z. Khatir, S. Lefebvre and F. Saint-Eve, "Experimental and Numerical Investigations on Delayed Short-Circuit Failure Mode of Single Chip IGBT Devices,” Microelectronics Reliability, 47, 2007, 422-428.

[5] Castellazzi, M. Ciappa, W. Fichtner, M. Piton and M. Mermet-Guyennet, "A Study of the Threshold Voltage Suitability as an Application-Related Reliability Indicator for Planar-Gate Non-Punch Through IGBTs," Microelectronics Reliability, 47, 2007, 1713-1718.

[6] J. Baliga, “Temperature Behaviour of Insulated Gate Transistor Characteristics,” Solid State Electronics, 28, 1985, 289-297.

[7] Maiga, H. Toutah, B. Tala-Ighil and B. Boudart, "Behaviour of Punch-Through and Non-Punch-Through Insulated Gate Bipolar Transistors Under High Temperature Gate Bias Stress," Proceedings of the IEEE International Symposium on Industrial Electronics, 2, 2004, 1035-1040.

[8] T. Tewksbury III and H. Lee, “Characterization, Modelling, and Minimization of Transient Threshold Voltage Shifts in MOSFETs,” IEEE Journal of Solid State Circuits, 29, 1994, 239-252,

[9] J.H. Stathis, "Percolation Models for Gate Oxide Breakdown,” in Journal of Applied Physics, 86, 1999, 5757-5766

Addresses of the authors

Nishad Patil, Diganta Das and Michael Pecht, Center for Advanced Life Cycle Engineering (CALCE), University of Maryland, College Park, MD 20742, USA, pecht@calce.umd.edu

Kai Goebel, NASA Ames Research Center, Moffett Field, CA 94035, USA, kai.goebel@nasa.gov 